\title{
A presentation for the Thompson sporadic simple group
}

\author{
George Havas, Leonard H. Soicher and Robert A. Wilson
}

\begin{abstract}
We determine a presentation for the Thompson sporadic simple group Th. The proof of correctness of this presentation uses a coset enumeration of $143,127,000$ cosets. In the process of our work, we also determine presentations for ${ }^{3} D_{4}(2),{ }^{3} D_{4}(2): 3, G_{2}(3): 2$, and $C_{T h}(2 A)$ (of shape $2_{+}^{1+8} \cdot A_{9}$ ). We also provide, via the internet, matrices generating $T h$ and satisfying our presentation.
\end{abstract}

1991 Mathematics Subject Classification: primary 20D08, 20F05, 20-04.

\section{Introduction}

Presentations for many sporadic simple groups and their automorphism groups have been published. For example, see [PS97] for $M_{11}, M_{12}, M_{22}, M_{23}, M_{24}$, $J_{1}, J_{2}, H S, M c L, H e, S u z, O^{\prime} N, \mathrm{Co}_{1}, \mathrm{Co}_{2}, \mathrm{Co}_{3}, \mathrm{Fi}_{22}, \mathrm{Fi}_{23}, \mathrm{Fi}_{24}$; see [HM69] for $J_{3}$, [Wei91] for $R u$, [BN97] for $H N: 2$, [JW96,HS99] for $L y$, [SW88,Iva92] for $J_{4}$, and [Iva99] for $B$ and $M$. Indeed, until now, the Thompson group $T h$ was the only sporadic simple group $S$ for which there was no published presentation for $S$ or Aut $(S)$. In this paper, we apply computational group theory in various ways to determine a presentation for $T h(\cong$ Aut $(T h))$. In the process, we also determine presentations for ${ }^{3} D_{4}(2)$, ${ }^{3} D_{4}(2): 3, G_{2}(3): 2$, and $C_{T h}(2 A)$. We also provide, via the internet, matrices generating $T h$ and satisfying our presentation.

Throughout, we use ATLAS notation [ATLAS] for conjugacy classes and group structures. The proof of correctness of the presentation for $T h$ uses a coset enumeration of 143,127,000 cosets, first completed during the Conference.

\section{The Thompson group}

In the monster group $M$, the centralizer of an element in conjugacy class $3 C$ is of shape $3 \times T h$, where $T h$ is the Thompson (sporadic simple) group. The group 
Th has order $90,745,943,887,872,000=2^{15} \cdot 3^{10} \cdot 5^{3} \cdot 7^{2} \cdot 13.19 .31$. The maximal subgroups of $T h$ have been completely determined (see [Wil88,Lin89,Lin91]), the largest being ${ }^{3} D_{4}(2): 3$ of index $143,127,000$. The next largest maximal subgroup is the Dempwolff group $2^{5} \cdot L_{5}(2)$, of index $283,599,225$. The Thompson group contains just one conjugacy class of involutions, and the centralizer of an element in this class is a maximal subgroup of shape $2_{+}^{1+8} \cdot A_{9}$. There are exactly three conjugacy classes of elements of order 3 in $T h$, and the normalizer $N(3 A)$ of the subgroup generated by an element in the smallest such class is a maximal subgroup of shape $\left(3 \times G_{2}(3)\right): 2$.

\section{The presentations}

Theorem 3.1. Consider the group $T$ presented by generators $a, b, c, d, e, s, t, u$ and relators $(1)-(6)$ below:

$$
\begin{gathered}
a^{2}, b^{2}, c^{2}, d^{2}, e^{2},(a b)^{3},(a e)^{2},(b c)^{3},(b d)^{2},(b e)^{2}, a=(c d)^{4},(c e)^{2},(d e)^{3},(b c d e)^{8}, \\
s^{7},[s, a],[s, b],[s, c],(s d)^{2},[e, s]=e^{s^{3}}, \\
t^{3},[t, a],[t, b],[t, c],[t, d],[t, e], s^{t}=s^{2}, \\
u^{2}=a c,[u, a],[u, c],[u, e],\left(d e d^{u}\right)^{2},\left[u,(a c)^{b}\right]=e,\left[u^{d},(a c)^{b}\right]=u e(a c)^{b} u^{d} e c, \\
t^{u}=t^{-1}, \\
{\left[e, u^{s^{2}}\right], a c=(u s)^{3}=[u, s]^{4},\left(d u^{s^{2}}\right)^{4}=a c c^{d} c^{d e s^{-1}} c^{d e s^{2}} .}
\end{gathered}
$$

(Here, the equation $w_{1}=w_{2}$ denotes the relator $w_{1} w_{2}^{-1}$. The notation $w_{1}^{w_{2}}$ means $w_{2}^{-1} w_{1} w_{2}$, and $\left[w_{1}, w_{2}\right]$ means $\left.w_{1}^{-1} w_{2}^{-1} w_{1} w_{2}.\right)$

Then the following hold:

1. $T \cong T h$.

2. Generators $a, b, c, d, e, s$ together with relators $(1),(2)$ is a presentation for ${ }^{3} D_{4}(2)$.

3. Generators $a, b, c, d, e, s, t$ together with relators $(1),(2),(3)$ is a presentation for ${ }^{3} D_{4}(2): 3$.

4. Generators $a, b, c, d, e, u$ together with relators $(1),(4)$ is a presentation for $G_{2}(3): 2$. 
5. Generators a, $c, d, e, s, t, u$ together with the relators from $(1)-(6)$ not involving $b$ is a presentation for $C_{T h}(2 A)$.

Proof. Using the 248-dimensional GF(2)-matrix representation of $T h$ available from the ATLAS of Finite Group Representations [Wil98,W+99], we have constructed non-identity 248-dimensional $G F(2)$-matrices a, b, c, d, e, s, t, u $\in$ $T h$ satisfying all the relators $(1)-(6)$ above (in the obvious way). The interested reader can find these matrices in GAP-format (see [GAP4]) at

$$
\text { http : //www.mat.bham.ac.uk/atlas/gap/Th/ }
$$

and verify this. Later, we will see that these matrices generate $T h$. The determination of these matrices is described in Section 4.

Now the abstract generators $a, b, c, d, e$ together with the relators (1) is easily seen to be a presentation for the group $U_{3}(3): 2$ (this is essentially the same presentation as that for $U_{3}(3): 2$ which appears in [Soi92]). Indeed, the reader is encouraged to (at least) check that the group so presented has order 12,096. A coset enumeration shows that $\langle a, b, c, d, e\rangle$ has index 17,472 in the group $D$ presented by generators $a, b, c, d, e, s$ and relators (1), (2). Thus $D$, as well as its matrix group image

$$
\mathbf{D}:=\langle\mathbf{a}, \mathbf{b}, \mathbf{c}, \mathbf{d}, \mathbf{e}, \mathbf{s}\rangle,
$$

has order at most that of ${ }^{3} D_{4}(2)$. Moreover, the subgroup $\mathbf{D}$ of $T h$ contains $\langle\mathbf{a}, \mathbf{b}, \mathbf{c}, \mathbf{d}, \mathbf{e}\rangle \cong U_{3}(3): 2$ (as opposed to a proper quotient of this group, in which case the relators would imply $\mathbf{a}=1$, a contradiction), and also $\mathbf{D}$ contains $7^{2} \cong\left\langle\mathbf{b c c}^{\mathbf{d}}, \mathbf{s}\right\rangle$. From the maximal subgroup structure of $T h$ we conclude that the only possibility for $\mathbf{D}$ is ${ }^{3} D_{4}(2)$. Since the order of $D$ is bounded by that of ${ }^{3} D_{4}(2)$ as well as having $\mathbf{D}$ as a homomorphic image, we conclude that $D \cong{ }^{3} D_{4}(2)$, establishing Statement 2 of the theorem.

Given that $\mathbf{t}$ has order 3 and the relators (3), we conclude that $t$ is an element of order 3 in $T$, normalizing the subgroup $\langle a, b, c, d, e, s\rangle \cong{ }^{3} D_{4}(2)$ and centralizing its maximal subgroup $\langle a, b, c, d, e\rangle \cong U_{3}(3): 2$. Since no inner element of ${ }^{3} D_{4}(2)$ of order 3 has these properties, it follows that

$$
\mathbf{H}:=\langle\mathbf{D}, \mathbf{t}\rangle \cong{ }^{3} D_{4}(2): 3,
$$

and Statement 3 follows.

Now $\mathbf{H}$ is a maximal subgroup of $T h$, and $\mathbf{u} \notin \mathbf{H}$ since, by relator (5), $\mathbf{u}$ must invert t. Hence

$$
T h=\langle\mathbf{a}, \mathbf{b}, \mathbf{c}, \mathbf{d}, \mathbf{e}, \mathbf{s}, \mathbf{t}, \mathbf{u}\rangle,
$$

and we have that $T h$ is a homomorphic image of the group $T$ presented. Now the main Statement 1 of the theorem follows from the enumeration of $143,127,000$ cosets of $H:=\langle a, b, c, d, e, s, t\rangle$ in $T$. This enumeration is described in Section 5 . 
We now tackle Statement 4. The matrix $\mathbf{u}$ is in the normalizer of $\mathbf{t}$, with $\mathbf{u}$ inverting t. Hence

$$
\mathbf{G}:=\langle\mathbf{a}, \mathbf{b}, \mathbf{c}, \mathbf{d}, \mathbf{e}, \mathbf{u}\rangle \leq N_{T h}(\mathbf{t}) \cong\left(3 \times G_{2}(3)\right): 2,
$$

and it is easy to see that $\mathbf{G} \cong G_{2}(3): 2$ (with the full normalizer of $\langle\mathbf{t}\rangle$ being $\langle\mathbf{G}, \mathbf{t}\rangle)$. A coset enumeration shows that $\langle a, b, c, d, e\rangle$ has index 702 in the group presented by generators $a, b, c, d, e, u$ and relators (1), (4), establishing Statement 4.

Finally, we prove Statement 5. It is easily deduced from relators $(1)-(3)$ that $a$ commutes with $c, d, e, s$, and $t$. Then a coset enumeration shows that $\langle a, c, d, e, s, t\rangle$ has index 819 in $H=\langle a, b, c, d, e, s, t\rangle$, establishing that $\langle a, c, d, e, s, t\rangle=C_{H}(a) \cong 2_{+}^{1+8}: L_{2}(8): 3 \cong\langle\mathbf{a}, \mathbf{c}, \mathbf{d}, \mathbf{e}, \mathbf{s}, \mathbf{t}\rangle$. Since $[\mathbf{u}, \mathbf{a}]=1$ and $\mathbf{u} \notin \mathbf{H}$, we have that

$$
\mathbf{C}:=\langle\mathbf{a}, \mathbf{c}, \mathbf{d}, \mathbf{e}, \mathbf{s}, \mathbf{t}, \mathbf{u}\rangle=C_{T h}(\mathbf{a}) .
$$

Now let $C$ be the group presented by generators $a, c, d, e, s, t, u$ together with the relators from $(1)-(6)$ not involving $b$. It is easy to show, using coset enumeration, that in $C, L:=\langle d, e, s, t\rangle \cong L_{2}(8): 3$, and then a coset enumeration of the 61,440 cosets of $L$ in $C$ completes the proof of 5 .

We now outline (roughly) how our presentation for $T h$ was derived. We started by determining the presentation above for $H=\langle a, b, c, d, e, s, t\rangle \cong$ ${ }^{3} D_{4}(2): 3$, starting with the known presentation for $U:=\langle a, b, c, d, e\rangle \cong U_{3}(3): 2$, and using the subgroup structure of ${ }^{3} D_{4}(2): 3$ given in [ATLAS] to determine the further generators and relators. In $H, t$ is an outer element of order 3 centralized by $U$, and in $T h$, we can extend $U$ to $G_{2}(3): 2$ by an element $u$ inverting $t$. Up to automorphisms of $G_{2}(3): 2$ there is just one way to identify the elements $a, b, c, d, e$. Calculating in a permutation representation of $G_{2}(3): 2$, we identified these elements, chose an outer element $u$ centralizing $a$ and satisfying other short relators, and then determined a presentation for $G_{2}(3): 2$ on generators $a, b, c, d, e, u$. For this, the GAP4 function PresentationViaCosetTable was very helpful in determining the crucial relation $\left[u^{d},(a c)^{b}\right]=u e(a c)^{b} u^{d} e c$, to make correct the subgroup $\left\langle a, c,(a c)^{b}, c^{d}, e, u, u^{d}\right\rangle \cong 2^{3} \cdot L_{3}(2) .2$ in $G_{2}(3): 2$. Defining relators for $C_{T h}(a) \cong 2_{+}^{1+8} \cdot A_{9}$ on generators $a, c, d, e, s, t, u$ were more difficult to obtain, although starting with $\langle d, e, s, t\rangle \cong L_{2}(8): 3$, we determined defining relators modulo the normal $2_{+}^{1+8}$ (which is the normal closure of $c$ in $\langle a, c, d, e, s, t\rangle)$. To exactly determine defining relators for $C_{T h}(a)$, we then constructed the matrices generating $T h$, and satisfying all the relators $(1)-(5)$ above, and then deduced the relators (6).

Some of our relators for $T \cong T h$ are known to be redundant, but are included for clarity or to aid coset enumeration. We also remark that, in $T$, $\left\langle a, c,(a c)^{b}, c^{d}, e, s, t, u, u^{d}\right\rangle$ is isomorphic to the Dempwolff group $2^{5} \cdot L_{5}(2)$. 


\section{Determining matrices in $T h$ satisfying the presentation}

Inside the Thompson group, a copy of ${ }^{3} D_{4}(2): 3$ can easily be found in a random search of subgroups generated by an involution and a $3 C$-element. To find $3 \times U_{3}(3): 2$, the centralizer of a $3 C$-element in ${ }^{3} D_{4}(2): 3$, we first found two elements, of orders 21 and 12 , powering up to two different $3 C$-elements, and then in the group generated by these two 3-elements, found an element conjugating one to the other. (This could be done either by a random search, or by pure thought, since the group generated by these elements turned out to be $2^{3}: 7: 3$.) We thus obtain elements of orders 21 and 12 powering up to the same $3 C$-element, and generating the whole of its centralizer, $3 \times U_{3}(3): 2$. This $3 C$-element may be taken as $\mathbf{t}^{ \pm 1}$.

We then look for $\mathbf{a}, \mathbf{b}, \mathbf{c}, \mathbf{d}, \mathbf{e}$ satisfying the given presentation for $U_{3}(3): 2$. We first found an outer involution, which we can take to be e, and used standard methods to find its centralizer $2 \times S_{4}$. There are two subgroups isomorphic to $S_{4}$ in here, one of which is inside $U_{3}(3)$. We take $\mathbf{a}, \mathbf{b}, \mathbf{c}$ to be the transpositions (12), (23), (34) of the latter. It remains to find $\mathbf{d}$, which is unique up to conjugation by e. We do this by searching in the centralizer of another involution, such as a (or b). Since this centralizer is small, this does not take long.

The next step is to find $\mathbf{s}$ inside ${ }^{3} D_{4}(2): 3$. Now $\mathbf{s}$ is an element of order 7 commuting with $\langle\mathbf{a}, \mathbf{b}, \mathbf{c}\rangle \cong S_{4}$, so we work inside the normalizer of the foursgroup $\left\langle\mathbf{a c},(\mathbf{a c})^{\mathbf{b}}\right\rangle$, which is a group of shape $2^{2} \cdot\left[2^{9}\right] .\left(7 \times S_{3}\right)$. We take any element $\mathbf{s}_{0}$ of order 7 in this group, and then $\mathbf{s}_{1}=\mathbf{s}_{0}\left[\mathbf{b c}, \mathbf{s}_{0}\right]$ commutes with $\mathbf{b c}$, so $\mathbf{s}$ is a suitable power of $\mathbf{s}_{1}$. Indeed, we may take $\mathbf{s}=\mathbf{s}_{1}$ without loss of generality. At this point we can use the relation $\mathbf{s}^{\mathbf{t}}=\mathbf{s}^{2}$ to determine which central element of $3 \times U_{3}(3): 2$ is $\mathbf{t}$, and which is $\mathbf{t}^{-1}$.

Finally we must find $\mathbf{u}$, which we do inside the full centralizer $2_{+}^{1+8} \cdot A_{9}$ of a. This centralizer is generated by $\mathbf{c}, \mathbf{d}, \mathbf{e}, \mathbf{s}, \mathbf{t}$ and one other element, found by standard methods. We chop the representation to find a constituent which represents $A_{9}$, and convert everything to the natural permutation representation where we can calculate easily. We are looking for an element which commutes with e, and inverts $\mathbf{t}$. In the quotient $A_{9}$, there are just three involutions with these properties, but only one satisfies the relation $(\mathbf{u s})^{3}=1$. If $\mathbf{u}_{0}$ is any lift of this element in $2_{+}^{1+8} \cdot A_{9}$, then $\mathbf{u}_{1}=\mathbf{u}_{0}\left[\mathbf{t}, \mathbf{u}_{0}\right]$ inverts $\mathbf{t}$.

We now have $N(\mathbf{t}) \cong\left(3 \times G_{2}(3)\right): 2$ generated by $\mathbf{t}, \mathbf{a}, \mathbf{b}, \mathbf{c}, \mathbf{d}$, e and $\mathbf{u}_{1}$, but $\mathbf{u}_{1}$ still does not satisfy all the required relations, because we have not yet specified precisely which preimage of the element of $A_{9}$ to take. Inside $G_{2}(3): 2$ generated by $\mathbf{a}, \mathbf{b}, \mathbf{c}, \mathbf{d}, \mathbf{e}$ and $\mathbf{u}_{1}$ we find that the normalizer of the fours-group $\langle\mathbf{a}, \mathbf{c}\rangle$ is generated by bacb, $\mathbf{d c d}$, e and $\mathbf{u}_{1}$, and we search in this small group for the required element $\mathbf{u}$. 


\section{The large coset enumeration}

The enumeration to determine $|T: H|$ requires the definition of more than 140 million cosets. Thus this step requires substantial resources. Recent descriptions of relevant aspects of coset enumeration are given in [Sim94,HR]. Of particular importance here is that the space required (memory locations) for a standard coset enumeration to complete is at least the maximum number of cosets defined times the number of columns in the coset table. The number of columns in the coset table is the number of involutary group generators plus twice the number of noninvolutary generators.

In some circumstances (eg, see [HS99]) this means we need to consider finding presentations with small generating sets. In our case here the given presentation for $T$ leads to eleven columns, which is not too many in the context of memory resources available on some supercomputers. Our enumerations were performed on an SGI Origin 2000 computer which has 16 gigabytes of memory, and we were able to obtain access to some 8 gigabytes. This is enough to store over 180 million cosets with the given presentation.

For difficult coset enumeration problems there is sometimes the need for substantial experimentation to determine how to complete an enumeration with given resources (see [HR]). However our experience with the various subgroups of $T$ discussed in this paper led us to believe that the enumeration in this case would be relatively easy, and so it turned out.

Using the ACE3 coset enumerator [ACE], with group presentation and subgroup generators exactly as in this paper we can readily compute the 143,127,000 cosets of $H$ in $T$.

The ACE3 enumerator has a large number of parameters, with a wide choice of settings and enumeration styles (see [ACE]). We have completed the enumeration using various strategies without undue difficulty. Felsch-based enumerations seem best. They complete in as little as about $62 \mathrm{cpu}$ hours. For example, with the styles Hard, Felsch:0, Felsch:1 and Sims:3 in each case the maximum number of cosets defined is the index. The total numbers Hard Felsch:1 Felsch:0 Sims:3 are: $145,251,396 \quad 151,273,730 \quad 155,354,391 \quad 240,046,124$.

The HLT style seems less attractive, using a maximum of 179,631,904 cosets and a total of 473,745,756 in an enumeration given 8 gigabytes of memory.

\section{Acknowledgements}

The work of the first author was supported by the Australian Research Council. We are grateful to The University of Queensland's High Performance Computing Unit for access to the Origin 2000 supercomputer. 


\section{References}

[BN97] T. Breuer and S. Norton, Improvements to the ATLAS, Appendix 2 of: An ATLAS of Brauer Characters, (by C. Jansen, K. Lux, R. Parker and R. Wilson), Clarendon Press, Oxford, 1995, 297-327.

[ATLAS] J.H. Conway, R.T. Curtis, S.P. Norton, R.A. Parker and R.A. Wilson, An ATLAS of Finite Groups, Clarendon Press, Oxford, 1985.

[GAP4] The GAP Group, Lehrstuhl D für Mathematik, RWTH Aachen, Germany and School of Mathematical and Computational Sciences, U. St. Andrews, Scotland, GAP - Groups, Algorithms, and Programming, Version 4, 1999. GAP is available at http://www-gap.dcs.st-and.ac.uk/〜gap/.

[ACE] G. Havas and C. Ramsay, Coset enumeration: ACE version 3, 1999. Available as http://www.csee.uq.edu.au/ ${ }^{2}$ havas/ace3.tar.gz.

[HR] G. Havas and C. Ramsay, Experiments in coset enumeration, These proceedings.

[HS99] G. Havas and C.C. Sims, A presentation for the Lyons simple group, in: Computational Methods for Representations of Groups and Algebras, Progress in Mathematics 173, Birkhäuser, Basel, 1999, 241-249.

[HM69] G. Higman and J. McKay, On Janko's simple group of order 50,232,960, Bull. London Math. Soc. 1 (1969), 89-94.

[Iva92] A.A. Ivanov, A presentation for $J_{4}$, Proc. London Math. Soc. 64 (1992), 369-396.

[Iva99] A.A. Ivanov, Geometry of Sporadic Groups I: Petersen and Tilde Geometries, Cambridge University Press, 1999.

[JW96] C. Jansen and R.A. Wilson, The minimal faithful 3-modular representation for the Lyons group, Comm. Algebra 24 (1996), 873-879.

[Lin89] S.A. Linton, The maximal subgroups of the Thompson group, J. London Math. Soc., II 39 (1989), 79-88.

[Lin91] S.A. Linton, Correction to "The maximal subgroups of the Thompson group", J. London Math. Soc., II 43 (1991), 253-254.

[PS97] C.E. Praeger and L.H. Soicher, Low Rank Representations and Graphs for Sporadic Groups, Cambridge University Press, 1997.

[Sim94] C.C. Sims, Computation with Finitely Presented Groups, Cambridge University Press, 1994.

[Soi92] L.H. Soicher, On simplicial complexes related to the Suzuki sequence graphs, in: Groups, Combinatorics and Geometry (M.W. Liebeck and J. Saxl, eds), LMS Lecture Note Series 165, Cambridge University Press, 1992, 240-248.

[SW88] G. Stroth and R. Weiss, Modified Steinberg relations for the group $J_{4}$, Geom. Dedicata 25 (1988), 513-525.

[Wei91] R. Weiss, A geometric characterization of the groups $M_{12}, H e$ and $R u, \mathrm{~J}$. Math. Soc. Japan 43 (1991), 795-814. 
[Wil88] R.A. Wilson, Some subgroups of the Thompson group, J. Austral. Math. Soc. (A) 44 (1988), 17-32.

[Wi198] R.A. Wilson, An ATLAS of sporadic group representations, in: The ATLAS 10 Years On (R.T. Curtis and R.A. Wilson, eds), LMS Lecture Note Series 249, Cambridge University Press, 1998, 261-273.

[W+99] R.A. Wilson et al., An ATLAS of Finite Group Representations, http://www.mat.bham.ac.uk/atlas/, 1999.

George Havas

Centre for Discrete Mathematics and Computing

Department of Computer Science and Electrical Engineering

The University of Queensland

Queensland 4072, Australia

havas@csee.uq.edu.au

Leonard H. Soicher

School of Mathematical Sciences

Queen Mary and Westfield College

Mile End Road, London E1 4NS, UK

L.H.Soicher@qmw.ac.uk

Robert A. Wilson

School of Mathematics and Statistics

The University of Birmingham

Edgbaston, Birmingham B15 2TT, UK

R.A.Wilson@bham.ac.uk 